https://helda.helsinki.fi

\title{
Screening of HELQ in breast and ovarian cancer families
}

\author{
Pelttari, Liisa M.
}

2016-01

Pelttari , L M , Kinnunen , L , Kiiski , J I, Khan , S , Blomqvist , C , Aittomaki , K \&

Nevanlinna , H 2016 , ' Screening of HELQ in breast and ovarian cancer families ' , Familial

Cancer , vol. 15 , no. 1 , pp. 19-23 . https://doi.org/10.1007/s10689-015-9838-4

http://hdl.handle.net/10138/223861

https://doi.org/10.1007/s10689-015-9838-4

publishedVersion

Downloaded from Helda, University of Helsinki institutional repository.

This is an electronic reprint of the original article.

This reprint may differ from the original in pagination and typographic detail.

Please cite the original version. 


\title{
Screening of $H E L Q$ in breast and ovarian cancer families
}

\author{
Liisa M. Pelttari ${ }^{1} \cdot$ Laura Kinnunen $^{1} \cdot$ Johanna I. Kiiski $^{1} \cdot$ Sofia Khan $^{1}$ • \\ Carl Blomqvist $^{2} \cdot$ Kristiina Aittomäki $^{3} \cdot$ Heli Nevanlinna ${ }^{1}$
}

Published online: 8 September 2015

(C) Springer Science+Business Media Dordrecht 2015

\begin{abstract}
Several high and moderate risk alleles have been identified for breast and ovarian cancer predisposition and most of them encode proteins that function in DNA repair. A prospective candidate for breast and ovarian cancer susceptibility is the HELQ helicase that has a role in the resolution of DNA interstrand cross-links. HELQ interacts with the RAD51 paralog complex BCDX2. Two components of the complex, RAD51C and RAD51D, increase the risk of ovarian cancer especially, and the other two, RAD51B and XRCC2 have been associated with breast cancer risk. To investigate the role of $H E L Q$ in cancer predisposition, we screened the gene for germline variation in 185 Finnish breast or ovarian cancer families and performed haplotype analyses for 1517 breast cancer cases, 308 ovarian cancer cases, and 1234 population controls using five common polymorphisms at the $H E L Q$ gene locus. No truncating mutations were identified among the families. One putatively pathogenic missense mutation c.1309A $>\mathrm{G}$ was identified but no additional carriers were observed in the subsequent genotyping of 332 familial
\end{abstract}

Electronic supplementary material The online version of this article (doi:10.1007/s10689-015-9838-4) contains supplementary material, which is available to authorized users.

Heli Nevanlinna

heli.nevanlinna@hus.fi

1 Department of Obstetrics and Gynecology, University of Helsinki and Helsinki University Hospital, Biomedicum Helsinki, P.O. Box 700, 00029 Helsinki, Finland

2 Department of Oncology, University of Helsinki and Helsinki University Hospital, P.O. Box 180, 00029 Helsinki, Finland

3 Department of Clinical Genetics, University of Helsinki and Helsinki University Hospital, P.O. Box 160, 00029 Helsinki, Finland breast or ovarian cancer patients. Furthermore, the haplotype distribution did not differ between breast or ovarian cancer cases and population controls. Our results indicate that $H E L Q$ is not a major breast and ovarian cancer susceptibility gene in the Finnish population. However, we cannot rule out rare risk-variants in the Finnish or other populations and larger datasets are needed to further assess the role of $H E L Q$ especially in ovarian cancer predisposition.

Keywords $H E L Q \cdot$ Breast cancer · Ovarian cancer · DNA repair

\section{Introduction}

Large numbers of susceptibility loci for both breast and ovarian cancer have been identified yet the currently known risk alleles explain less than half of the excess familial risk. Thus, more risk genes and variants are likely to exist. The most important known susceptibility genes are $B R C A 1$ and BRCA2 that confer high life-time risks of breast and ovarian cancer while mutations in moderatepenetrance genes such as CHEK2, ATM, BRIP1, and $P A L B 2$ confer milder risks and, in addition, a vast amount of low-risk loci have been identified for both breast and ovarian cancer [1-3]. Most of the susceptibility genes function in DNA repair and specifically, some of them have a role in the Fanconi Anemia (FA) pathway that is required for the repair of DNA interstrand cross-links (ICLs). The FA pathway is initiated by FANCM which is part of the multi-protein FA core complex [4]. The FA core complex monoubiqitinates FANCD2 and FANCI which then localize to DNA lesions and coordinate ICL repair with the downstream FA proteins. Biallelic mutations in the 
downstream FA genes BRCA2, PALB2, BRIP1, and $R A D 51 C$ cause FA subtypes FA-D1, FA-N, FA-J, and FA$\mathrm{O}$, respectively, whereas heterozygous mutations in these genes increase the risk of breast or ovarian cancer. The downstream FA proteins are important for the homologous recombination (HR) repair of DNA double-strand breaks that are created during the ICL resolution. Central players in HR are RAD51 and the five RAD51 paralogs RAD51B, RAD51C, RAD51D, XRCC2, and XRCC3. The paralogs form two major complexes together: the BCDX2 complex containing RAD51B, RAD51C, RAD51D, and XRCC2; and the CX3 complex containing RAD51C and XRCC3 [5].

We have previously identified Finnish founder mutations in the RAD51 paralogs RAD51C and RAD51D that increase the risk of ovarian cancer $[6,7]$ and recently, we identified a nonsense mutation in the FANCM gene among Finnish breast cancer patients conferring an increased risk especially for triple-negative breast cancer [8]. These results highlight the isolated Finnish founder population as an excellent resource for the identification of new susceptibility genes and alleles as the bottlenecks have enriched certain low-frequency variants while other rare variants have disappeared. Thus, in isolated founder populations the majority of mutations in specific genes may be explained by few recurrent mutations whereas in out-bred populations large numbers of very rare mutations in each gene may be present.

The HELQ helicase was recently shown to interact with the BCDX2 complex as well as with the DNA damageresponsive kinase ATR and HELQ-deficient human and mouse cells were found to be sensitive for ICL-inducing agents suggesting a role for HELQ in the processing of ICLs [9, 10]. Helq deficient mice are more susceptible to tumors than wildtype controls with ovarian and pituitary tumors being the most frequent tumor types in female Helq ${ }^{\Delta C / \Delta C}$ mice [10]. Interestingly, also $\mathrm{Helq}^{+/ \Delta C}$ female mice developed ovarian pathologies, but with less severe phenotypes than in the homozygous mutant mice, indicating haploinsufficiency for Helq. Moreover, the Helq ${ }^{\Delta C / \Delta C}$ mice resemble mouse models of FA and the silencing of $H E L Q$ in human cells resulted in similar phenotypes. The role of HELQ in ICL processing and tumor suppression as well as its interaction with the RAD51 paralogs makes $H E L Q$ an attractive candidate for breast and ovarian cancer susceptibility gene.

To evaluate the role of HELQ in breast and ovarian cancer predisposition and to identify putative recurrent founder mutations, we screened 185 Finnish breast or ovarian cancer families for germline variation in the $H E L Q$ gene. We also studied the association of common variation in the HELQ gene with breast and ovarian cancer risk by haplotype analysis in a large case-control dataset.

\section{Materials and methods}

\section{Subjects}

Two unselected cohorts of breast cancer patients, including 79 and $87 \%$ of all consecutive, newly diagnosed breast cancer cases during the collection periods, were collected at Helsinki University Hospital Department of Oncology in 1997-1998 and $2000(\mathrm{n}=884)$ [11, 12] and Department of Surgery in 2001-2004 $(n=986)$ [13] and additional familial breast and ovarian cancer patients were ascertained at Helsinki University Hospital Departments of Oncology and Clinical Genetics $[13,14]$. The HELQ gene was analyzed in 185 breast or ovarian cancer families (including 113 breast, 68 breast-ovarian, and four ovarian cancer families) tested negative for $B R C A 1$ and $B R C A 2$ mutations as previously described [15-17]. Altogether 174 patients (including 164 breast, four breast-ovarian, and six ovarian cancer cases) were screened by Sanger sequencing and 11 families were screened by exome sequencing of 24 patients. The identified c.1309A $>\mathrm{G}$ mutation was subsequently screened in 332 familial breast or ovarian cancer patients and a haplotype analysis was performed for 1517 breast cancer cases, 308 ovarian cancer cases, and 1234 population controls that were genotyped as part of the iCOGS study [1, 2]. The population controls were healthy female blood donors from the same geographic region. Informed consent was obtained from all individual participants and the study was approved by the Ethics committee of the Helsinki University Hospital.

\section{Sequencing}

The coding regions and the exon-intron boundaries of the HELQ gene were Sanger sequenced in germline DNA samples of 174 patients. The DNA was amplified with PCR (Supplementary Table 1) and the PCR products were cleaned with ExoSAP-IT treatment (Affymetrix) and sequenced with ABI BigDyeTerminator 3.1 Cycle Sequencing kit (Life Technologies). The capillary sequencing was performed at the Institute for Molecular Medicine Finland (FIMM), University of Helsinki, using 3730xl DNA Analyzer (Life Technologies). The sequence chromatograms were examined with Variant Reporter 1.0 software (Life Technologies). Exome sequencing for 24 patients from 11 families was performed as previously described [8].

\section{Genotyping}

The identified c.1309A $>\mathrm{G}$ mutation was genotyped in germline DNA samples of 332 patients by TaqMan realtime PCR using custom TaqMan SNP Genotyping assays 
and TaqMan Genotyping MasterMix (Life Technologies). The PCR was performed in 7500 Fast Real-Time PCR system and the results were analyzed with TaqMan Genotyper software (Life Technologies).

\section{Statistical methods and bioinformatics}

The pathogenicity of the identified missense variants was predicted with SIFT, PolyPhen, and MutationTaster [18]. The haplotype analysis was performed with PHASE v2.1.1 software [19, 20]. Five polymorphisms (rs4693623, rs4693625, rs13141136, rs17006826, and rs4693626), spanning 41,230 base pairs within the HELQ gene locus, were used for the haplotype analysis with genotypes defined in iCOGS chip genotyping $[1,2]$. The distribution of the haplotypes was compared between cases and controls. To test the association of each polymorphism with breast and ovarian cancer, odds ratios (OR) and $95 \%$ confidence intervals (CI) were estimated by logistic regression using the $\mathrm{R}$ version 3.0.2 statistical software (http://www.r-project.org/).

\section{Results}

The screening of the HELQ gene in 185 breast or ovarian cancer families revealed seven sequence changes within the exons, including two synonymous changes rs13141136 and rs7665103 and five non-synonymous missense variants (Table 1). Two rare variants, c.796A $>\mathrm{G}$ and c.1309A $>\mathrm{G}$, were identified in the exome sequencing. The c.796A $>\mathrm{G}$ variant is detected once in the non-Finnish European population of the Exome Aggregation Consortium (ExAC) dataset $(\mathrm{n}=33$ 370) [Exome Aggregation Consortium (ExAC), Cambridge, MA; http://exac.broadinstitute.org (February 2015)] but has not been detected in the Finnish population $(n=3307)$ whereas the c.1309A $>\mathrm{G}$ is not observed in any of the ExAC populations. As the c. $1309 \mathrm{~A}>\mathrm{G}$ variant has not been reported before and was predicted to be pathogenic by SIFT, PolyPhen, and MutationTaster, it was selected for further genotyping. The rest of the missenses were predicted to be benign changes and were detected at comparable frequencies as in the ExAC Finnish population. The c.1309A $>\mathrm{G}$ missense was subsequently genotyped in additional 332 familial breast or ovarian cancer patients but no further carriers were identified. The two exome-sequenced relatives of the mutation carrier did not harbor the mutation.

To study the association of common variation in the $H E L Q$ gene with breast and ovarian cancer risk, we performed haplotype analyses for 1517 breast cancer cases, 308 ovarian cancer cases, and 1234 population controls using five SNPs within the HELQ gene locus. Altogether 10 haplotypes were predicted among the samples set (Table 2). The haplotype distribution did not differ between breast cancer cases and controls $(P=0.28)$ or between ovarian cancer cases and controls $(P=0.63)$. We also tested the association of each variant with breast and ovarian cancer risk but none of them showed significant association $(P>0.05)$ (Table 3$)$.

\section{Discussion}

To our knowledge, this is the first study to screen the $H E L Q$ gene for germline variation in breast and ovarian cancer families. HELQ is an attractive candidate for cancer susceptibility gene given its role in DNA repair and ICL resolution and interaction with the ovarian cancer susceptibility genes $R A D 51 C$ and $R A D 51 D[9,10]$.

We screened 185 Finnish breast or ovarian cancer families for germline variation in the HELQ gene but no truncating mutations were identified. A putatively pathogenic missense mutation c.1309A $>\mathrm{G}$, not present in the

Table 1 Non-synonymous missense changes detected in the sequencing of the $H E L Q$ gene

\begin{tabular}{|c|c|c|c|c|c|c|c|c|c|c|c|}
\hline $\begin{array}{l}\text { DNA } \\
\text { change }\end{array}$ & $\begin{array}{l}\text { Protein } \\
\text { change }\end{array}$ & rs-number & AA & $\mathrm{Aa}$ & aa & MAF & SIFT & PolyPhen & MutationTaster & $\begin{array}{l}\text { ExAC } \\
\text { FIN }\end{array}$ & $\begin{array}{l}\text { ExAC } \\
\text { NFE }\end{array}$ \\
\hline c. $53 \mathrm{~A}>\mathrm{G}$ & p.(Asn18Ser) & rs141700135 & 184 & 1 & 0 & 0.003 & Tolerated & Benign & Polymorphism & 0.010 & 0.006 \\
\hline c. $106 \mathrm{C}>\mathrm{T}$ & p.(Pro36Ser) & rs138939487 & 179 & 6 & 0 & 0.016 & Tolerated & Benign & Polymorphism & 0.020 & 0.004 \\
\hline c. $796 \mathrm{~A}>\mathrm{G}$ & p.(Lys266Glu) & na & 184 & 1 & 0 & 0.003 & Tolerated & $\begin{array}{l}\text { Possibly } \\
\text { damaging }\end{array}$ & Disease causing & na & 0.00002 \\
\hline c. $916 \mathrm{G}>\mathrm{A}$ & p.(Val306Ile) & rs1494961 & 38 & 97 & 50 & 0.532 & Tolerated & Benign & Polymorphism & 0.518 & 0.489 \\
\hline c. $1309 \mathrm{~A}>\mathrm{G}$ & p.(Thr437Ala) & na & 184 & 1 & 0 & 0.003 & Damaging & $\begin{array}{l}\text { Probably } \\
\text { damaging }\end{array}$ & Disease causing & na & na \\
\hline
\end{tabular}

$A A, A a$, a $a$ numbers of common homozygotes, heterozygotes, and rare homozygotes, respectively, MAF minor-allele frequency in this study, ExAC FIN MAF in the ExAC Finnish population, ExAC NFE MAF in the ExAC non-Finnish European population 
Table 2 Haplotypes detected in the sample set with frequencies among breast and ovarian cancer cases and population controls

\begin{tabular}{|c|c|c|c|c|c|c|}
\hline \multirow[t]{2}{*}{ Haplotype } & \multicolumn{3}{|c|}{ BC cases versus controls: $P=0.28$} & \multicolumn{3}{|c|}{ OC cases versus controls: $P=0.63$} \\
\hline & $\begin{array}{l}\text { Haplotype } \\
\text { count }\end{array}$ & $\begin{array}{l}\text { Frequency } \\
\text { BC cases }(\%)\end{array}$ & $\begin{array}{l}\text { Frequency } \\
\text { controls } \\
(\%)\end{array}$ & $\begin{array}{l}\text { Haplotype } \\
\text { count }\end{array}$ & $\begin{array}{l}\text { Frequency } \\
\text { OC cases (\%) }\end{array}$ & $\begin{array}{l}\text { Frequency } \\
\text { controls } \\
(\%)\end{array}$ \\
\hline GAGGG & 1775 & 30.91 & 32.39 & 1015 & 31.70 & 32.48 \\
\hline AGAAG & 1762 & 32.12 & 30.48 & 976 & 33.38 & 30.53 \\
\hline AGAAA & 834 & 15.35 & 14.80 & 470 & 16.63 & 14.81 \\
\hline AAGGG & 551 & 10.91 & 10.42 & 299 & 10.02 & 10.35 \\
\hline GGAAG & 317 & 6.50 & 6.31 & 169 & 5.26 & 6.22 \\
\hline GAGAG & 255 & 4.06 & 5.27 & 147 & 2.57 & 5.26 \\
\hline GGAAA & 3 & 0.08 & 0.12 & 3 & 0.25 & 0.15 \\
\hline AAGAG & 2 & 0.06 & 0.08 & 1 & 0.03 & 0.09 \\
\hline GGGGG & 2 & 0 & 0.05 & 3 & 0.16 & 0.08 \\
\hline GAAAA & 1 & 0 & 0.04 & 1 & 0 & 0.04 \\
\hline
\end{tabular}

The polymorphisms included in the analysis are described in Table 3

$B C$ breast cancer, $O C$ ovarian cancer

Table 3 Polymorphisms included in the haplotype analysis and their association with breast and ovarian cancer risk

\begin{tabular}{lllllllll}
\hline rs-number & HGVS & $\mathrm{MAF}_{\mathrm{BC}}$ & $\mathrm{MAF}_{\mathrm{OC}}$ & $\mathrm{MAF}_{\mathrm{ctrl}}$ & $\mathrm{OR}_{\mathrm{BC}}(95 \% \mathrm{CI})$ & $P_{\mathrm{BC}}$ & $\mathrm{OR} \mathrm{OC}_{(95 \% \mathrm{CI})} P_{\mathrm{OC}}$ \\
\hline rs4693623 & $\mathrm{c} .3199-3055 \mathrm{~A}>\mathrm{G}$ & 0.42 & 0.40 & 0.44 & $0.90(0.81-1.00)$ & 0.051 & $0.84(0.70-1.01)$ & 0.059 \\
rs4693625 & $\mathrm{c} .1465+800 \mathrm{C}>\mathrm{T}$ & 0.46 & 0.44 & 0.48 & $0.91(0.82-1.02)$ & 0.093 & $0.86(0.72-1.03)$ & 0.099 \\
rs13141136 & $\mathrm{c} .1036 \mathrm{~T}>\mathrm{C}$ & 0.46 & 0.44 & 0.48 & $0.91(0.82-1.01)$ & 0.089 & $0.86(0.72-1.03)$ & 0.093 \\
rs17006826 & c. $1013-269 \mathrm{~A}>\mathrm{G}$ & 0.42 & 0.42 & 0.43 & $0.95(0.86-1.06)$ & 0.402 & $0.97(0.81-1.16)$ & 0.728 \\
rs4693626 & $\mathrm{c} .1012+1386 \mathrm{~A}>\mathrm{G}$ & 0.15 & 0.17 & 0.15 & $1.03(0.89-1.20)$ & 0.650 & $1.15(0.91-1.45)$ & 0.252 \\
\hline
\end{tabular}

The polymorphisms are presented in the same order as in the haplotypes in Table 2

$M A F_{B C}, M A F_{O C}$, and $M A F_{c t r l}$ minor-allele frequencies among breast cancer cases, ovarian cancer cases, and population controls, respectively, $O R_{B C}$ and $O R_{O C}$ odds ratios for breast and ovarian cancer, $C I$ confidence interval, $P_{B C}$ and $P_{O C} p$ values for breast and ovarian cancer

ExAC database, was detected in one breast cancer patient but it was not observed among additional 332 familial breast or ovarian cancer cases in the subsequent genotyping nor in two breast cancer relatives of the mutation carrier. Another rare variant c.796A $>\mathrm{G}$, observed only once in the whole ExAC database, was detected once in the screening of the HELQ gene but this variant was predicted to be tolerated by SIFT and was not studied further. In addition, three benign missense changes were detected at similar frequencies as in the Finnish population of the ExAC database. Of note, one of the detected common missense variants, rs1494961 in the exon 2, has been associated with risk for oral cavity and pharynx cancers in a genome-wide association study [21].

To study the common variation in HELQ and the association with breast and ovarian cancer, we performed haplotype analyses for 1517 breast cancer cases, 308 ovarian cancer cases, and 1232 healthy population controls.
The haplotype distribution did not differ between the breast or ovarian cancer cases and population controls suggesting that common variation in $H E L Q$ does not play a major role in breast or ovarian cancer predisposition.

Despite the growing number of identified susceptibility loci, large portion of the familial risk of breast and ovarian cancer remains unexplained. The remaining may be explained by several common low-risk variants as well as very rare higher-risk alleles. Our results from the sequencing of the breast and ovarian cancer families and the haplotype analysis suggest that $H E L Q$ does not significantly contribute to breast and ovarian cancer susceptibility in the Finnish population. However, we cannot rule out rare risk-variants in the Finnish or other populations. The observed c.1309A $>\mathrm{G}$ missense variant may be unique for the carrier and its contribution to breast cancer susceptibility remains uncertain. As the number of ovarian cancer cases was small in our sequencing analysis, the 
contribution of rare $H E L Q$ mutations to ovarian cancer predisposition cannot be excluded and studies with larger sample sizes are needed to better evaluate the role of $H E L Q$ in ovarian cancer susceptibility. Given the diverse tumortypes present in $\mathrm{Helq}^{\Delta \mathrm{C} / \Delta C}$ mice [10] studies also in others cancer types are warranted to evaluate the role of $H E L Q$ mutations in other malignancies.

\section{Conclusions}

The absence of deleterious HELQ mutations among familial breast and ovarian cancer patients and the similar distribution of haplotypes between breast and ovarian cancer cases and population controls indicate that $H E L Q$ does not significantly contribute to breast cancer predisposition in the Finnish population. However, larger datasets of ovarian cancer patients are needed to better assess the role of HELQ in ovarian cancer predisposition. Furthermore, we cannot exclude the presence of very rare mutations in $H E L Q$ that may confer an increased risk of breast cancer.

Acknowledgments We thank research nurse Irja Erkkilä for her help with collecting the patient samples and data, Dr. Ralf Bützow for the ovarian cancer patient samples, Prof. Douglas Easton and Manjeet Bolla for the iCOGS genotyping data, and the Finnish Cancer Registry for the cancer diagnostic data. This study was supported by the Helsinki University Hospital Research Fund, the Academy of Finland (266528), the Sigrid Juselius Foundation, the Finnish Cancer Society, and the Nordic Cancer Union and for LMP by the Finnish Cultural Foundation.

\section{Compliance with ethical standards}

Conflict of interest The authors declare that they have no conflict of interest.

\section{References}

1. Michailidou K, Hall P, Gonzalez-Neira A et al (2013) Largescale genotyping identifies 41 new loci associated with breast cancer risk. Nat Genet 45:353-361. doi:10.1038/ng.2563

2. Pharoah PD, Tsai YY, Ramus SJ et al (2013) GWAS metaanalysis and replication identifies three new susceptibility loci for ovarian cancer. Nat Genet. doi:10.1038/ng.2564

3. Mavaddat N, Antoniou AC, Easton DF, Garcia-Closas M (2010) Genetic susceptibility to breast cancer. Mol Oncol 4:174-191. doi:10.1016/j.molonc.2010.04.011

4. Kim H, D'Andrea AD (2012) Regulation of DNA cross-link repair by the Fanconi anemia/BRCA pathway. Genes Dev 26:1393-1408. doi:10.1101/gad.195248.112

5. Suwaki N, Klare K, Tarsounas M (2011) RAD51 paralogs: roles in DNA damage signalling, recombinational repair and tumorigenesis. Semin Cell Dev Biol 22:898-905. doi:10.1016/j. semcdb.2011.07.019

6. Pelttari LM, Heikkinen T, Thompson D et al (2011) RAD51C is a susceptibility gene for ovarian cancer. Hum Mol Genet 20:3278-3288. doi:10.1093/hmg/ddr229

7. Pelttari LM, Kiiski J, Nurminen R et al (2012) A Finnish founder mutation in RAD51D: analysis in breast, ovarian, prostate, and colorectal cancer. J Med Genet 49:429-432. doi:10.1136/jmed genet-2012-100852

8. Kiiski JI, Pelttari LM, Khan S et al (2014) Exome sequencing identifies FANCM as a susceptibility gene for triple-negative breast cancer. Proc Natl Acad Sci USA 111:15172-15177. doi:10.1073/pnas.1407909111

9. Takata K, Reh S, Tomida J, Person MD, Wood RD (2013) Human DNA helicase HELQ participates in DNA interstrand crosslink tolerance with ATR and RAD51 paralogs. Nat Commun 4:2338. doi:10.1038/ncomms3338

10. Adelman CA, Lolo RL, Birkbak NJ et al (2013) HELQ promotes RAD51 paralogue-dependent repair to avert germ cell loss and tumorigenesis. Nature 502:381-384. doi:10.1038/nature12565

11. Syrjäkoski K, Vahteristo P, Eerola H et al (2000) Populationbased study of BRCA1 and BRCA2 mutations in 1035 unselected Finnish breast cancer patients. J Natl Cancer Inst 92:1529-1531

12. Kilpivaara O, Bartkova J, Eerola H et al (2005) Correlation of CHEK2 protein expression and c.1100delC mutation status with tumor characteristics among unselected breast cancer patients. Int J Cancer 113:575-580. doi:10.1002/ijc.20638

13. Fagerholm R, Hofstetter B, Tommiska J et al (2008) $\mathrm{NAD}(\mathrm{P}) \mathrm{H}$ :quinone oxidoreductase $1 \mathrm{NQO} 1 * 2$ genotype $(\mathrm{P} 187 \mathrm{~S})$ is a strong prognostic and predictive factor in breast cancer. Nat Genet 40:844-853

14. Eerola H, Blomqvist C, Pukkala E, Pyrhonen S, Nevanlinna H (2000) Familial breast cancer in southern Finland: how prevalent are breast cancer families and can we trust the family history reported by patients? Eur J Cancer 36:1143-1148

15. Vehmanen P, Friedman LS, Eerola H et al (1997) Low proportion of BRCA1 and BRCA2 mutations in Finnish breast cancer families: evidence for additional susceptibility genes. Hum Mol Genet 6:2309-2315

16. Vahteristo P, Bartkova J, Eerola H et al (2002) A CHEK2 genetic variant contributing to a substantial fraction of familial breast cancer. Am J Hum Genet 71:432-438. doi:10.1086/341943

17. Vahteristo P, Eerola H, Tamminen A, Blomqvist C, Nevanlinna $\mathrm{H}$ (2001) A probability model for predicting BRCA1 and BRCA2 mutations in breast and breast-ovarian cancer families. $\mathrm{Br} \mathrm{J}$ Cancer 84:704-708. doi:10.1054/bjoc.2000.1626

18. Schwarz JM, Rodelsperger C, Schuelke M, Seelow D (2010) MutationTaster evaluates disease-causing potential of sequence alterations. Nat Methods 7:575-576. doi:10.1038/nmeth0810-575

19. Stephens M, Smith NJ, Donnelly P (2001) A new statistical method for haplotype reconstruction from population data. Am J Hum Genet 68:978-989. doi:10.1086/319501

20. Stephens M, Scheet P (2005) Accounting for decay of linkage disequilibrium in haplotype inference and missing-data imputation. Am J Hum Genet 76:449-462. doi:10.1086/428594

21. Babron MC, Kazma R, Gaborieau V, McKay J, Brennan P, Sarasin A, Benhamou S (2014) Genetic variants in DNA repair pathways and risk of upper aerodigestive tract cancers: combined analysis of data from two genome-wide association studies in European populations. Carcinogenesis 35:1523-1527. doi:10. 1093/carcin/bgu075 